\title{
The multigap resistive plate chamber as time-of-flight detector for the STAR experiment at RHIC
}

\author{
José Lamas $\mathrm{V}^{\mathrm{a}, \mathrm{b}^{*}}$ \\ ${ }^{a}$ Rice university, Bonner Nuclear Lab, Houston, USA \\ ${ }^{b}$ CERN, EP division, Geneva, Switzerland
}

\begin{abstract}
The Multigap Resistive Plate Chamber (MRPC) is a suitable candidate for the Time-of-Flight system for the STAR ${ }^{1}$ experiment at RHIC ${ }^{2}$ at the BNL ${ }^{3}$. A time resolution of $50 \mathrm{ps}$ with an efficiency of $98 \%$ has been measured with MRPCs composed of 6 gas gaps of $220 \mu \mathrm{m}$. Results obtained during the year 2000 are reported here. 102002 CSI. All rights reserved

Keywords: Resistive plate chambers; Time-of-Flight; Particle Identification; Gaseous detector; MRPC; STAR.

\section{Resumen}

La cámara de multiplacas resistivas (MRPC) es un detector candidato apropiado para el sistema de Tiempo-de-Vuelo del experimento STAR en el colisionador RHIC del laboratorio BNL. La MRPC presentada aquí esta compuesta de 6 espacios de gas de $220 \mu \mathrm{m}$, con ella se ha obtenido una resolución en la medida del tiempo de $50 \mathrm{ps}$ con una eficiencia de detección de $98 \%$. Los resultados presentados aquí corresponden a los tests realizados durante el año 2000. @ 2002 CSI. Todos los derechos reservados
\end{abstract}

Palabras Clave: Cámara de placas resistivas; Tiempo-de-Vuelo; Identificación de partículas; Detector gaseoso; MRPC; STAR

\section{Introduction}

The STAR experiment at RHIC consists of several detector sub-systems in a large solenoidal magnet of 0.5 Tesla. STAR is a large experiment that was designed to measure the thousands of particles per event at mid-rapidity expected in central Au+Au collisions at $100 \mathrm{GeV} / \mathrm{N} /$ beam.

\footnotetext{
"Corresponding author. e-mail: jose.lamas.valverde@cern.ch

${ }^{1}$ Soleinoidal Tracker at Rhic, www.bnl.gov/rich/star.htm!

2 relativistic Heavy ion Collider; www.bnl.gov/rhic/

${ }^{3}$ Brookhaaven National Laboratory; www.bnl.gov'
}

The physics potential of the STAR detector will be enhanced with the improved particle identification provided by a segmented Time-of-Flight (TOF) system. To achieve this, the TOF system must measure the time of flight of as many particles as possible in the central region with a time resolution better than $100 \mathrm{ps}$ for a flight path from $2.2 \mathrm{~m}$ to $2.9 \mathrm{~m}$ depending on pseudorapidity, $\eta$. A highly segmented and cylindrical detector immediately surrounding the $4.3 \mathrm{~m}$ 
long STAR Time Projection Chamber (TPC) can meet this requirement. Particle identification (PID) in the STAR TPC is done using the fact that pions, kaons, and protons are not minimum-ionizing in the energy range of the STAR acceptance and thus exhibit different energy losses while traversing these detectors. In PID via Time-of-Flight, the absolute time interval between the occurrence of an event and the arrival of a particle at a specific location of the TOF system is measured. Tracking information from the TPC provides the momentum of a particle, and a total path length from the collision vertex to the measurement location. The total path length so measured by the TPC and the time-of-flight lead to a velocity, $\beta$, for each track, which, when compared to the track momentum, predicts a mass for each track. If the time can be measured to better than $-100 \mathrm{ps}$, then such analysis allow the direct particle identification of hadrons with high efficiency to significantly higher momenta than possible from TPC $\mathrm{dE} / \mathrm{dx}$ alone.

This TOF detector must be capable of operating in a 0.5 Tesla magnetic field. In the original design of the TOF detector for STAR, scintillators were proposed. These were to be arranged in 120 trays and cover an area of $60 \mathrm{~m}^{2}$. Each individual tray is $2.4 \mathrm{~m}$ long, $21 \mathrm{~cm}$ wide and $8.5 \mathrm{~cm}$ deep ( $\mathrm{z} \times$ phi $\times \mathrm{r}$ ). Although this scintillator detector will certainly work, the cost of mesh-dynode phototubes required for operation in the magnetic field is prohibitive. Since 1998 the Rice University TOF group has been developing Multigap Resistive Plate Chambers (MRPC) suitable for the STAR experiment, and following the decision of the ALICE experiment at CERN to build a MRPC system for the Time-of-Flight ${ }^{2}$, a major effort was launched to develop these devices from the year 2000 in collaboration with the ALICE TOF group. We built and tested many prototypes of MRPCs at the Proton Synchrotron (PS) at CERN. The goal of these tests was to build a device with a time resolution better than 100 ps and with cell and detector dimensions appropriate for the STAR detector at RHIC..

\section{Detector description}

The Multigap Resistive Plate Chamber (MRPC) was developed in 1996 [3,4]. A resistive plate chamber (RPC) is a parallel plate chamber (PPC) with resistive electrodes instead of metallic electrodes used in PPCs. The utility of resistive electrodes is to quench the streamers so that they do not initiate a spark breakdown. By choosing a suitable gas mixture, operation of RPCs either in avalanche or streamer mode is possible [5]. The MRPC is a resistive plate chamber with intermediate resistive plates, which create a series of gas gaps. A cross-section view of the chamber here reported is shown in Fig.1. For these tests we built a series of chambers using glass resistive plates. Electrodes are applied to the outer surfaces of the two outer glass plates. A strong electric field is generated in each subgap by applying a high voltage across these external electrodes. All the internal glass sheets are electrically floating; they take the voltage as defined by electrostatics. We used float glass plates with a bulk resistivity of around $5 \times 10^{12} \Omega \cdot \mathrm{cm}$. The glass plates are kept parallel by using $240 \mu \mathrm{m}$ diameter nylon fishing-line. The electrodes are made of graphite tape with surface resistivity of $\mathbf{5 0 0}$ $\mathrm{k} \Omega / \mathrm{square}$ which covers the entire active area. We use a non-flammable gas mixture, which contains $90 \%$ of tetrafluoro-ethane $\left(\mathrm{C}_{2} \mathrm{H}_{2} \mathrm{~F}_{4}\right), 5 \%$ of iso-Butane and $5 \%$ of $\mathrm{SF}_{6}$. We report here results obtained with chambers with six gas gaps of $220 \mu \mathrm{m}$. The active area of all these prototypes is $6.5 \mathrm{~cm} \times 19.5 \mathrm{~cm}$. The signal is read out with a $2 \times 6$ array of copper pickup pads, each with an area of $31 \mathrm{~mm} \times 30$ $\mathrm{mm}$. The distance between pads is $3 \mathrm{~mm}$. We also studied pads of larger area, some results are presented in section 3 .

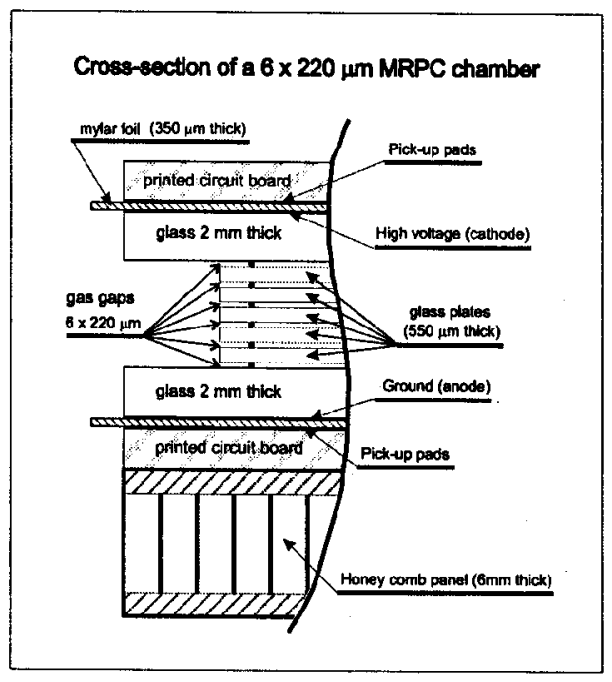

Fig 1. Cross-section of the $6 \times 220 \mu \mathrm{m}$ MRPC prototype for TOF

\section{Test beam result}

The purpose of these tests at the PS beam facility was to measure the time and efficiency performance of the MRPC. The tests were done with a beam spot of $\sim 4 \mathrm{~cm}^{2}$ and an intensity of $500 \mathrm{~Hz} / \mathrm{cm}^{2}$ during the PS spill of $350 \mathrm{~ms}$. The operation of these chambers is in avalanche mode.

The signal from the pads was amplified by using a fast current amplifier Maxim 3760. This amplifier was connected to a custom built discriminator based on the AD96685 comparator. Figure 2a shows a typical pulse height distribution obtained with an operation voltage of $15.5 \mathrm{kV}$, in the middle of the plateau curve. The equivalent mean charge for that distribution is around $500 \mathrm{fC}$. Fig. $2 \mathrm{~b}$ shows the Time-to-Amplitude (TA) correlation used for the offline correction for slewing [6]. 

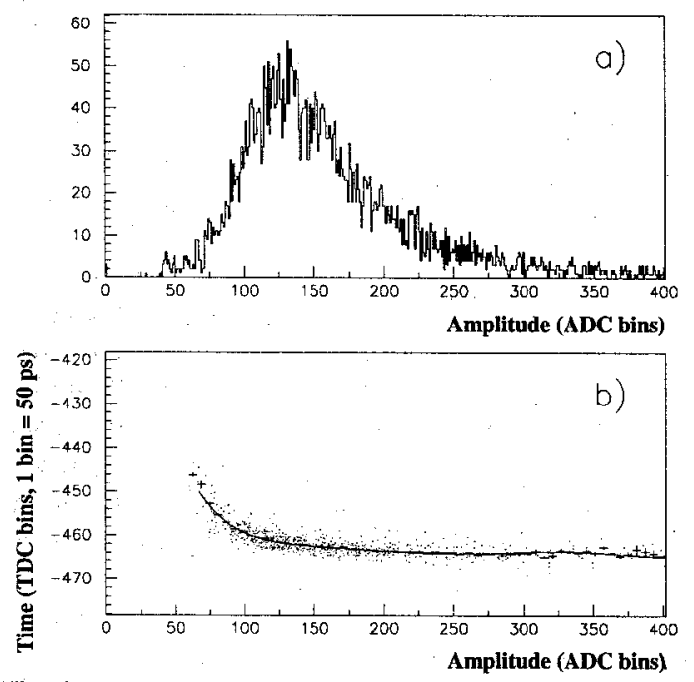

Fig. 2. a) A typical pulse height distribution from a six gaps multigap RPC at $15.5 \mathrm{kV}$. b) Time-to-Amplitude correlation. The vertical scale is the RPC time minus the average time of two reference scintillators. Superimposed is the curve fitted to the data.

In Fig. 3 the histograms show the time resolution a) before (raw time) and b) after Time-to-Amplitude correction. The distributions are fitted with Gaussians. Comparing the two distributions one observes that the overall time resolution improves and we obtain a variance, $\sigma$, of $94 \mathrm{ps}$ and $57 \mathrm{ps}$ for the uncorrected and TA corrected distributions. After subtracting the mean time jitter introduced by two scintillator counters. (4. PhotoMultipliers), we obtain $90 \mathrm{ps}$ and $50 \mathrm{ps}$ respectively.

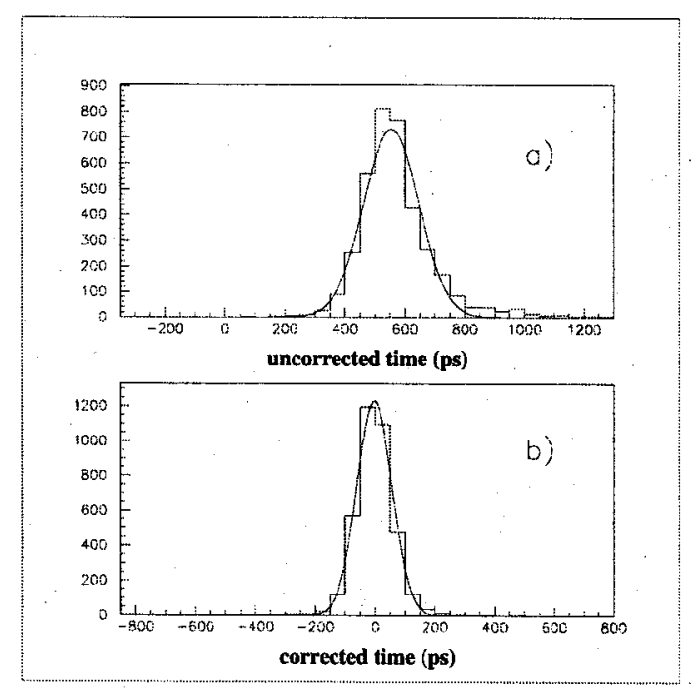

Fig.3. a) The raw time, and b) the Time-to-Amplitude corrected time distributions for a 6 gaps MRPC at $15.5 \mathrm{kV}$.
The efficiency and the TA corrected time resolution versus voltage are shown in Fig. $4 \mathrm{a}$. An efficiency of $98 \%$ has been measured over a $2 \mathrm{kV}$ region with a time resolution of $55 \mathrm{ps.} \mathrm{We} \mathrm{have} \mathrm{also} \mathrm{studied} \mathrm{the} \mathrm{effect} \mathrm{of}$ larger pad size on time resolution (TA corrected). We built a $1 \times 6$ array by combining two pads from the $2 \times 6$ array into a single pad. The resulting pad size is $65 \mathrm{~mm} \times 30 \mathrm{~mm}$. The signal is picked up from one of the edges of the rectangular pads. The results are shown in Fig. 4b. The time resolution at a point $50 \mathrm{~mm}$ from the pickup edge is nearly the same as that of the $31 \mathrm{~mm} \times 30 \mathrm{~mm}$ pads of the $2 \times 6$ array. The time resolution for positions closer to the pickup edge is 8 to 15 ps worse.

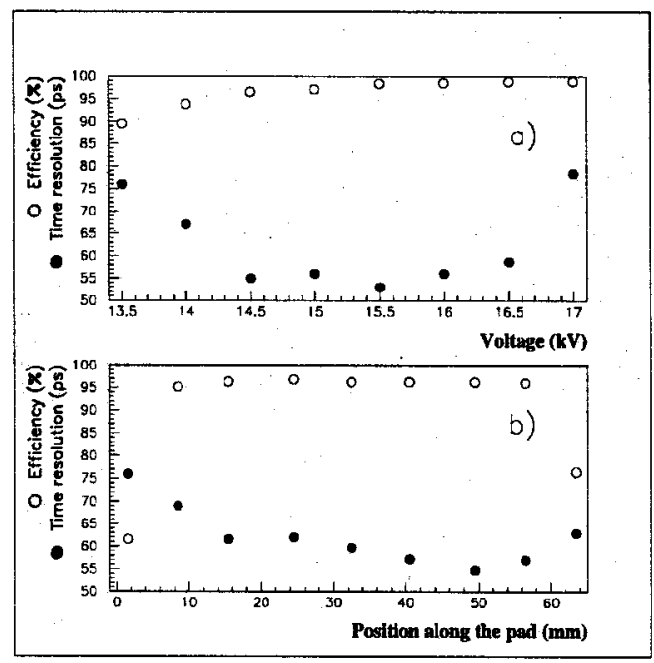

Fig. 4 a) Efficiency and corrected time resolution ( $\sigma$ ) versus voltage for a 6 gas gaps MRPC. The pad area is $31 \mathrm{~mm} \times 30 \mathrm{~mm}$. b) Efficiency and corrected time resolution $(\sigma)$ versus position along the pad. The voltage applied was $15.0 \mathrm{kV}$. The pad size is $65 \mathrm{~mm} \times 30 \mathrm{~mm}$. The signal pickup edge of the pad is at $0 \mathrm{~mm}$ in the horizontal scale.

\section{Conclusions}

The results of the tests performed at the Proton Synchrotron at CERN with the MRPC here described clearly show that efficiencies in excess of $95 \%$ and time resolutions of order of 50 ps can be achieved with this device, and this over a relatively broad high voltage plateau. These excellent results make MRPCs a very suitable candidate for the Time-of-Flight system for the STAR experiment at RHIC. 


\section{Acknowledgement}

I am deeply indebted to the ALICE TOF group. The R\&D program to develop MRPCs for STAR has been carried out inside the ALICE R\&D program. I am grateful particularly to Dr. D. Hatzifotiadou and Dr. M.C.S. Williams of the INFN Bologna and CERN/EP-Division, for their technical assistance during the test beam and for enriching discussions. Special thanks also to the CERN staff who run the PS accelerator so well.

\section{References}

[1]. W.J. Llope et al. Proposal for a 'Single Tray' Time-of-Flight Patch for STAR, 1998. STAR Time-of-Flight Documents.
[2]. ALICE Technical Design Report of the Time of Flight System, CERN / LHCC 2000-12 ALICE TDR 8, 16 February 2000.

[3]. E. Cerron Zeballos, I. Crotty, D. Hatzifotiadou, J. Lamas Valverde, S. Neupane, M.C.S. Williams and A. Zichichi, Nuclear Instruments and Methods in Physics Research A 374, (1996) p. 132-135.

[4]. J. Lamas Valverde. Doctoral Thesis: Development of the resistive plate chamber (RPC) and the multi-gap RPC: gaseous detectors for muon trigger for Large Hadron Collider experiments. Strasbourg University, France. March 1997 (language: French).

[5]. E. Cerron Zeballos, I. Crotty, D. Hatzifotiadou, J. Lamas Valverde, M.C.S. Williams and A. Zichichi, Nucl. Instr. Methods A 396, (1997) p. 93-102.

[6]. T. Sugitate, Y. Akiba, S. Hayashi, Y. Miake and S. Nagamiya and M. Torikoshi, Nucl. Instr. Methods A 249, (1986) p. 354. 